\title{
Enjeux de valeurs dans une correspondance électronique entre un jeune de cité et un sociologue
}

Values at stake in an electronic correspondence between a suburban youth and a sociologist

Jean-Claude Guerrini

\section{OpenEdition}

Journals

\section{Édition électronique}

URL : http://journals.openedition.org/aad/959

DOI : 10.4000/aad.959

ISSN : 1565-8961

Éditeur

Université de Tel-Aviv

Référence électronique

Jean-Claude Guerrini, «Enjeux de valeurs dans une correspondance électronique entre un jeune de cité et un sociologue », Argumentation et Analyse du Discours [En ligne], 5 | 2010, mis en ligne le 20 octobre 2010, consulté le 23 septembre 2019. URL : http://journals.openedition.org/aad/959 ; DOI 10.4000/aad.959

Ce document a été généré automatiquement le 23 septembre 2019.

\section{cc)}

Argumentation \& analyse du discours est mis à disposition selon les termes de la licence Creative Commons Attribution - Pas d'Utilisation Commerciale - Pas de Modification 4.0 International. 


\title{
Enjeux de valeurs dans une correspondance électronique entre un jeune de cité et un sociologue
}

Values at stake in an electronic correspondence between a suburban youth and a sociologist

\author{
Jean-Claude Guerrini
}

1 La lecture de Pays de malheur! Un jeune de cité écrit à un sociologue de Younès Amrani et Stéphane Beaud ${ }^{1}$, a suscité de nombreux commentaires ${ }^{2}$. Il ne s'agit pas, dans cet article, d'examiner la représentativité d'un témoignage, mais d'étudier la manière dont cette relation épistolaire par courriels construit une figure discursive qui, à la fois, éclaire et contredit l'image figée et stigmatisée du «mec de banlieue » enfermé dans des attitudes monolithiques et des partis pris indestructibles. Chacun des deux correspondants nourrit des attentes différentes à l'égard de son allocutaire, à partir d'univers de valeurs dont le contenu et les hiérarchisations se précisent peu à peu. Leur échange relève à la fois du dialogue intime, le sociologue tenant la place du confident, et d'une forme de partage, sur fond d'indignation commune contre les injustices sociales.

2 Cette correspondance n'aborde pas la question des valeurs de façon explicite. On pourrait même dire qu'elle en évite la thématique pour des raisons de fond. Situation courante qui justifie, en préambule, une mise au point théorique sur la notion de «relief axiologique». J'examinerai ensuite comment la correspondance permet d'affermir un cadre coopératif qui présuppose un accord sur les valeurs. Puis j'en viendrai aux aspects moins consensuels des échanges qui, même s'ils s'inscrivent dans ce dispositif coopératif, tendent à le déborder, en soulignant plus particulièrement les tensions axiologiques qui apparaissent parfois entre les interlocuteurs. 


\section{Valeurs, valorisation et interaction}

3 Rappelons tout d'abord brièvement quelles sont les principales manifestations de l'activité axiologique ${ }^{3}$ dans le discours. Se prononcer sur ce qui est bon, souhaitable ou préférable, et non pas sur le vrai, le valide ou le probable, suppose un garant qui ne soit ni une vérité ni un fait, mais une option concernant ce qui est digne d'estime, ce qui vaut devant un auditoire dont on cherche à recueillir l'adhésion, et qui constitue une règle pour l'action. Diverses possibilités, plus ou moins voyantes, s'offrent au locuteur.

\section{1. Le « relief axiologique»}

Des maîtres mots, généralement des substantifs, comme justice, loyauté, dignité, jouant le rôle de médiations, peuvent rappeler les accords censés garantir l'interaction : ils peuvent être invoqués ("au nom de la liberté ») ou convoqués de manière directe ou indirecte par une prise en charge plus discrète («ils sont morts dans la dignité »), par leur présupposition dans des lexèmes de catégories différentes (digne, dignement) ou par le recours à des antonymes (indignité, indigne). Ces maîtres mots (ou leurs contraires) assurent une présence explicite des normes du jugement. Ils peuvent donner lieu à des commentaires, à des définitions, à des argumentations en forme, et être ouvertement préconisés afin de produire un effet d'évidence ou de clarification ${ }^{4}$. Mais ils s'exposent, alors, à la contestation et à la critique. C'est ainsi que la locution " au nom de », censée autoriser une décision ou une prise de position, se trouve servir plus fréquemment une disqualification, la teneur de l'énoncé, mais aussi la posture énonciative d'invocation donnant prise à une remise en cause («Liberté, que de crimes on commet en ton nom !»).

5 Cela explique la faveur des procédures implicites qui permettent de jouer sur les accords présupposés ou sous-entendus. Dans Dire et ne pas dire (1972: 5), Oswald Ducrot souligne les bénéfices attendus de l'implicite : « on a fréquemment besoin, à la fois de dire certaines choses, et de pouvoir faire comme si on ne les avait pas dites, de les dire, mais de façon telle qu'on puisse refuser la responsabilité de leur énonciation ». Si l'on applique ces remarques au cas des valeurs, on peut parler d'un "relief axiologique" dont le locuteur peut jouer en fonction de son implication dans leur prise en charge. D'un ethos théâtral d'affichage des valeurs à un effacement des indices évaluatifs visant l'« objectivité ", les choix discursifs et rhétoriques peuvent varier et se combiner. Les choix lexicaux (adjectifs, noms, verbes, adverbes) jouent un rôle capital dans l'orientation argumentative en procédant à une modalisation plus ou moins discrète, parfois très localisée, des énoncés : un terme « objectif » ou portant une évaluation non axiologique, peut se trouver affecté d'une connotation valorisante ou dévalorisante dans le but de favoriser une orientation argumentative («commercial» peut être neutre, laudatif ou péjoratif). Cette possibilité d'infléchir les connotations habituelles rend difficile, voire impossible l'établissement de classes lexicales véritablement soustraites à la subjectivité évaluative et témoigne du tropisme qui porte à prendre parti, pour ou contre, en toute occasion. 


\section{2. Objets et interactants}

6 A cela il faut ajouter, dans une perspective pragmatique de prise en compte des pôles de l'interaction, que la « manie évaluative » affecte le statut des objets du discours et la place que se voient attribuer les interactants. Le Traité de l'argumentation de Chaïm Perelman et Lucie Olbrechts-Tyteca insiste sur le fait que, l'attention aux êtres étant limitée et contrainte par l'expérience, une hiérarchisation s'opère inévitablement :

C'est un donné psychologique qui, comme le montre Piaget, exerce une action dès le niveau de la perception : lors de la confrontation de deux éléments, par exemple un étalon fixe et des grandeurs variables auxquelles on le compare, ce sur quoi le regard est centré, ce qui est vu mieux ou plus souvent est, de ce seul fait, surévalué (Perelman \& Tyteca $1988: 156)$.

7 Selon cette logique comparative, l'orientation du discours argumentatif résulte en bonne part de la façon dont les objets du discours sont sélectionnés et affectés d'une " présence » qui témoigne de leur « importance et [de] leur pertinence dans le débat ». C'est ce qui explique que la valorisation (et son contraire la dévalorisation) qui associe présence et hiérarchisation constitue, dans le mouvement même de la mobilisation des valeurs, une notion centrale du Traité. La classification, qui est étroitement liée à l'activité verbale de nomination et de qualification, relève aussi d'un mécanisme élémentaire d'orientation : des catégories déjà constituées, "pré-jugées ", aident à se situer et à évaluer. C'est moins l'objet qui alors s'impose, par l'effet d'un contraste perceptif, qu'une opposition construite en fonction de laquelle le sujet pourra attribuer des places, valorisées ou non, aux objets, mais aussi à son interlocuteur et à lui-même. Le Traité de l'argumentation cite l'attitude du pauvre blanc qui se sent "valorisé » par l'opposition des noirs et des blancs, mais aussi cet autre cas relevé par Simone de Beauvoir dans Le deuxième sexe : «Une foi sincère, aide beaucoup la fillette à éviter tout complexe d'infériorité : elle n'est ni mâle ni femelle, mais une créature de Dieu $»^{5}$.

Pour celui qui est engagé dans une délibération intime, l'activité axiologique affecte l'image qu'il se fait de lui-même ; et, dans le cadre d'une interlocution, les places étant, dès la phase d'ouverture, distribuées, l'objectif consistera pour chacun des participants à consolider ou modifier, en même temps que les représentations et les valeurs, l'image de soi qu'il prétend assumer. C'est pourquoi il paraît indispensable, si l'on se donne pour but d'observer le rôle des valeurs dans un échange épistolaire à enjeu relationnel, de prendre en compte, en même temps que les garants axiologiques qui sont en jeu (des maitres-mots et/ou des personnages référents), les autres aspects de l'interlocution, les interactants eux-mêmes et les objets à l'égard desquels ils se déterminent - sans oublier la dynamique dans laquelle ils sont pris.

\section{Une correspondance fondée sur un projet coopératif}

Un jour de décembre 2002, un emploi-jeune de 28 ans d'origine marocaine, habitant dans l'agglomération lyonnaise, qui vient d'achever la lecture de $80 \%$ au bac...et après? Les enfants de la démocratisation scolaire, envoie un courriel à l'auteur. Il a relevé son adresse électronique sur le site de l'Ecole Normale Supérieure de la rue d'Ulm et veut lui exprimer son enthousiasme et sa gratitude :

Mercredi 11 décembre 2002

Cher monsieur,

Je me permets de vous écrire pour vous remercier. J'ai terminé votre enquête $80 \%$ 
au bac. C'est un livre qui m'a à la fois ému (j'ai souvent eu les larmes aux yeux) et mis en colère (contre moi-même). C'est incroyable à quel point les vies que vous avez décrites ressemblent à la mienne. D'ailleurs je dois dire que la lecture était parfois pénible car c'est dur de se voir dans un miroir. En tout cas, j'ai appris beaucoup sur ma vie et cela va sûrement m'être utile pour mon avenir. Maintenant j'essaie de faire de la «pub» pour votre livre auprès de mes amis d'enfance du quartier que j'ai quittés, mais c'est trop dur (trop de préjugés, passivité face aux problèmes...). J'essaie de leur faire comprendre que l'échec relatif dans lequel nous sommes n'est pas dépendant que de nous et que ce n'est pas non plus que de leur «faute », etc. (2005: 11). (79 sont de Stéphane Beaud, 108, beaucoup plus longs, de Younès Amrani), selon un rythme variable: de un à huit messages le même jour avec des périodes de plus grande fréquence. Parfois des conversations téléphoniques s'intercalent, mais très rarement, Stéphane Beaud exerçant une pression très ferme pour qu'il s'agisse d'un recours exceptionnel. Trois séances d'entretiens auront lieu en janvier, en mars et en juillet ${ }^{6}$. Younès n'a pas Internet chez lui et écrit de la bibliothèque municipale où il travaille. Beaud, qui prépare un nouveau livre et dont la charge d'enseignant-chercheur est importante, rédige des courriels dans l'urgence entre deux tâches professionnelles. Les deux hommes partagent, au moment où débute la correspondance, un socle de valeurs communes qui va permettre à Younès de se livrer à un profond travail d'anamnèse et le conduire à s'exposer, au point de mettre en danger cruellement sa propre image. Sur cette base, pourront s'exprimer des désaccords partiels et des efforts d'explicitation qui nous retiendront dans la troisième partie.

\section{2. Deux critères axiologiques cruciaux : l'« utile » et l'« intéressant »}

11 L'émotion et l'intérêt sont réciproques, chacun insistant sur l'utilité de la sociologie en quelque sorte prouvée par la rencontre du livre et du lecteur. En réponse à Younès qui affirme que le livre va lui être « utile », Beaud répond :

Cher Monsieur,

Votre lettre m'a beaucoup touché et bien sûr me conforte dans l'idée que la sociologie peut servir à quelque chose.

Un « « grand » $»^{7}$ conseil : reprenez votre licence d'histoire (il y a aussi le CNED) et passez dans deux ans le CAPES...

Younès est gratifié à l'idée de bénéficier de l'attention et des conseils d'un universitaire reconnu et sensible aux problèmes des jeunes qui lui ressemblent; le sociologue est intrigué de se trouver devant un lecteur d'exception, curieux et perspicace : d'où la récurrence du marqueur axiologique "intéressant» (Younès: "Vos articles avec M. Pialoux sont très intéressants "; Stéphane Beaud: "Merci pour ce début. C'est très, très intéressant»). Les axes de valorisation épistémique (« intéressant») et pragmatique («utile») se trouvent ainsi étroitement liés au cœur même de l'échange. Le sociologue vérifie le résultat de ses recherches antérieures et perçoit de possibles développements. Au bout de quelque temps, il montre les courriels échangés à ses amis chercheurs, envisage un article avec Younès, puis lui propose de publier leur correspondance, qui se poursuit avec, comme interlocuteurs virtuels, au-delà de sa propre personne, les lecteurs d'un livre futur 8 . Le livre parait en 2004 accompagné d'une postface dans laquelle le sociologue fournit une mise en contexte et un

Argumentation et Analyse du Discours, 5 | 2010 
commentaire de cette correspondance. Une édition de poche est publiée l'année suivante accompagnée d'une série de courriels reçus par les auteurs en réaction à Pays de Malheur! et d'une réponse du jeune bibliothécaire à ceux qui demandaient « ce que devenait Younès »: malgré les sollicitations des médias, Younès Amrani souhaite maintenir l'anonymat que la démarche sociologique lui a ménagé. Sous ce pseudonyme, le scripteur réalise la mise entre parenthèses de "l'être empirique » que l'analyse du discours s'efforce d'effectuer.

Les rapports de place, étroitement liés aux statuts, sont immédiatement installés et ne subiront que peu de modifications ${ }^{9}$ : le "jeune de cité », marié et père d'un enfant, Ismaël, mais assumant difficilement ce passage à la vie adulte, trouve auprès de ce sociologue qui intervient dans les médias ${ }^{10}$, un confident et un interlocuteur privilégié ; l'enseignant- chercheur est familier des problèmes rencontrés par ces étudiants de familles ouvrières récemment installées en France qui ne parviennent pas à dépasser le seuil de la première ou deuxième année de fac. D'accord sur la valeur du savoir ${ }^{11}$ et sur la légitimité de l'" ambition scolaire ", les deux correspondants s'engagent dans une entreprise d'élucidation à laquelle ils trouvent un intérêt différent mais complémentaire, et que le sociologue, caractérise en des termes assez hétérodoxes - l'énonciation hybride traduisant l'intensité de sa participation : «En fait, ce que l'on fait ensemble, c'est de contribuer à une espèce d'auto-analyse de votre parcours scolaire» (21). Mais si le cadre ainsi instauré les rapproche, les deux hommes, en revanche, sont aux antipodes quant au parcours de réussite. Le sociologue, constatant une fois de plus les effets du désenchantement engendré par des études longues chez des enfants d'ouvriers d'origine immigrée, encourage Younès à reprendre ses études et à s'insérer dans le monde enseignant. Mais, celui-ci, "remotivé » dans un premier temps par un livre qui donne sens à son itinéraire, butte contre un sentiment d'échec profondément ancré.

\section{3. Un ethos coopératif}

14 Le point de départ du récit de vie dans lequel s'engage Younès touche au cœur du dispositif de production de valeur, à l'articulation du « fait », de l'interprétation et des effets axiologiques qui en découlent. Dans son troisième courriel du 11 décembre 2002 (premier jour de la correspondance), le jeune bibliothécaire tient à signaler que la lecture de $80 \%$ au bac... bouleverse l'image d'un sociologue qui, en classe de seconde, a joué un rôle crucial dans sa trajectoire scolaire: Pierre Bourdieu. Les tableaux statistiques de corrélation entre origine sociale et réussite scolaire, présentés par son professeur d'Initiation économique et sociale, l'avaient scandalisé :

À la base, je pense, avec le recul que le prof avait de bonnes intentions mais, en fait, moi, je l'ai pris comme une attaque. Je me disais: qu'est-ce qu'il nous veut ce Bourdieu ! Il veut nous démoraliser ou quoi! En plus j'aimais pas du tout la manière avec laquelle le prof amenait ça parce que ça nous rabaissait devant les autres (style, on est pas à notre place ici, etc). [...] Bref, je me suis dit : il faut que j'en sache plus sur ce Bourdieu qui veut nous « descendre » (14).

Le lycéen relie spontanément sa place dans l'édifice social, sa valeur et la manière dont il peut envisager son parcours scolaire. Face à l'attitude distanciée du professeur de lycée qui croit pouvoir en rester à des données statistiques brutes, Younès se perçoit comme la cible d'une disqualification; il en est réduit à chercher dans les postures 
discursives du professeur, puis dans son comportement dans les conseils de classe, le sens qu'il convient de leur affecter.

Plus de dix ans après, la lecture de $80 \%$ au bac... produit un nouvel électro-choc : Younès se jette simultanément dans la lecture de Pierre Bourdieu, dont il comprend désormais la démarche, et dans un travail d'anamnèse avec Stéphane Beaud. Il y manifeste des aptitudes qui viennent contredire la représentation habituelle du " jeune de quartier " désinvolte, imprévisible et agressif : respectant ses «tours d'écriture » avec régularité, il se plie à la rigueur de l'enquête dont il est l'objet. Il s'efforce de répondre aux attentes de son correspondant en se conformant spontanément aux maximes du discours, s'inquiète de l'intérêt de ses propos, de leur densité, de leur qualité, ce qui prend parfois l'allure d'un échange conversationnel, comme dans cette « paire adjacente » du 9 janvier (les courriels se suivent immédiatement) :

Younès : - C'est précis?

Stéphane : - C'est très précis... (60).

17 La question de la pertinence revient souvent. Younès perçoit bien que ses textes sont « représentatifs »; mais apportent-ils un éclairage original ? : «PS : Que pensez-vous de "tout" ce que je vous raconte. Est-ce utile ? Vous avez dû rencontrer ça des tas de fois » (16). Le sociologue, de son côté, invite Younès à " creuser » tel ou tel aspect abordé trop rapidement et lui fournit des "pistes d'écriture »: thèmes (le service militaire, la religion, les rapports avec les filles), batteries de questions, demandes d'analyses.

Tout repose sur un pacte : «Je vous écris tout cela parce que j'ai vraiment confiance en vous (par rapport à vos livres, vos articles)» (19). Le "professeur» suggère des ouvrages dans un but pédagogique (grands classiques de la sociologie et de l'histoire, de Maurice Halbwachs ou de Marc Bloch, par exemple), fait part de ses propres découvertes (romans, articles de journaux); de son côté, le jeune bibliothécaire surprend l'universitaire par l'ambition de ses lectures passées ou en cours (Massignon et Corbin sur la religion musulmane, Questions de sociologie de Bourdieu).

Il y a également accord sur le registre des échanges, du moins pour l'essentiel. Le primat de la description, lié au parti pris d'explication causale d'un itinéraire, invite à une faible convocation de valeurs explicites. Il conduit plutôt à une relation des émotions ressenties (rage, fierté, honte) ou à des verdicts d'amélioration / dégradation ou d'acceptation / rejet, présupposant des valeurs restant implicites (justice, honnêteté et véracité, le plus souvent). Younès cherche à maîtriser sa tendance spontanée - «Il faut que je m'astreigne à comprendre et non à juger " (151). De son côté, Beaud ne répond pas aux sollicitations du jeune homme lorsque celui-ci anticipe une réprobation éventuelle concernant sa conduite ou ses opinions ("Désolé ", "Je dois vous choquer ", etc.) et cherche à provoquer un jugement explicite. Il enchaîne alors sur le contenu informatif et néglige d'apporter les évaluations attendues.

Cela n'empêche ni l'un ni l'autre de recourir à l'occasion à des termes fortement dépréciatifs ("scandaleux»71, "conneries» 89, etc.). Dans le cas de Younès, ils relèvent même du registre de l'insulte, la cible étant parfois sa propre personne ( J'étais une grosse pourriture de raciste qui refusait tout en bloc» 196). Le « relief axiologique » des courriels varie donc sensiblement. Par exemple, lorsque dans un élan enthousiaste, Younès déclare "Je souhaiterais qu'il puisse y avoir des gens vraiment désintéressés qui croient en l'humanité, à la justice, au partage, réellement des gens "purs" "; ou quand le sociologue cherche à identifier le système de valeurs du jeune homme en lui demandant franchement "Petite question: comment on repère une 
famille "bien"?» (148). Mais ce qui domine nettement, c'est une forte retenue dans l'expression explicite des jugements de valeur.

21 Il faut noter en revanche un trait caractéristique : l'usage fréquent des guillemets chez les deux participants, qui traduit une attention soutenue aux mots, une méfiance à l'égard des prétendues évidences du langage. La modalisation autonymique souligne en effet l'importance d'une notion ou d'une qualification. Stéphane Beaud rappelle, par exemple, une formule ou un qualificatif figurant dans l'échange: "votre trip “arabité" " (26), "tu étais comme tu dis dans ta phase "intransigeante" " (138). Fréquemment, les guillemets indiquent un commentaire implicite portant sur le statut axiologique problématique du lexème mentionné (quelques exemples : Younès - «leur faute " 11 , «ambition scolaire» 16, «beurs» 24, «dynamiques» 137; Beaud "l'intégration » 17 , «françaises de souche » 53, «conversion du regard " 86. Il peut s'agir de termes que le locuteur approuve, promeut; qu'il réprouve, dont il veut se démarquer; ou de mots dont il tient à montrer qu'il n'ignore pas leur dimension polémique. En tout cas, le déchiffrement des guillemets supposant une connivence (Maingueneau 2007), leur fréquence mesure la vigilance critique des deux participants, l'ampleur de leurs accords, mais aussi leur souci constant, même s'il est rarement explicité, de suspecter des valorisations inscrites dans le vocabulaire.

\section{4. Un maître mot : comprendre}

L'un et l'autre sont en fait animés par le mot " comprendre », qui semble leur avoir servi de «mot de passe » et de valeur commune, non sans quelque malentendu. Patrick Charaudeau, réfléchissant au rôle des valeurs, les considère comme des notions que les individus «se donnent en partage et qui servent de référence pour expliquer et juger leurs comportements et leurs actions » (2008: 23). Il les situe parmi les systèmes de croyance et « les imaginaires collectifs, plus ou moins flous, plus ou moins conscients qui témoignent de la façon dont les individus vivant en communauté se représentent les principes qui devraient présider à leur désir de "vivre ensemble" ». De ce flou, signalé par le Traité de l'argumentation (Perelman \& Tyteca 1992: 102) comme une condition de l'accord sur de grandes orientations du jugement ${ }^{12}$, Charaudeau fournit une présentation suggestive: une "perte sémantique au regard de la définition rigoureuse » se voit compensée par un " enrichissement sémantique » à force d'emplois dans des situations différentes » (comme dans le cas de " démocratie » et « éthique »).

C'est ce double mouvement qui semble affecter ici l'emploi du mot « comprendre » et de l'adjectif dérivé « compréhensif ». Dans la conclusion de $80 \%$ au bac..., Beaud mobilise en effet cet adjectif au sein d'un paragraphe polémique :

Aujourd'hui, lorsqu'on parle des problèmes de violence et d'insécurité dans les quartiers populaires et/ou des attitudes des jeunes de "cité » (majoritairement issus de l'immigration), il semble être à la mode, chez les responsables politiques, de dénoncer comme "irréalistes » toutes les formes de discours qui viseraient à expliquer ces attitudes, à les situer dans un contexte économique et social, à évoquer leur genèse sociale. Cette posture intellectuelle - « compréhensive ", pour le dire vite, qui a constitué l'apanage de la pensée de gauche et dont l'ordonnance de 1945 sur les mineurs est imprégnée, est de plus en plus assimilée à de l'angélisme ou à un progressisme de façade qui se montrerait sourd aux réalités du terrain et aux exaspérations légitimes devant la montée des «violences urbaines » et autres « incivilités » (303). 
L'adjectif « compréhensive » est mis entre guillemets par un auteur très au fait du rôle qu'il s'est vu attribuer, ainsi que le verbe «comprendre", dans les débats épistémologiques ${ }^{13}$ et les discours politiques. Younès, à qui la lecture du livre de Stéphane Beaud a tendu un miroir, est sensible, de son côté, à la dimension éthique du terme qui lui donne l'occasion de restaurer son image. Le verbe "comprendre" apparaît dès son premier courriel : « Je pense que beaucoup de profs, de flics et même de citoyens devraient lire votre livre pour ENFIN nous comprendre » (11). Et, fait significatif, ce mot, que l'on retrouve à de très nombreuses reprises chez les deux correspondants, vient au premier plan dans le dernier texte, détaché de l'ensemble, où Younès, à la demande de Beaud, fait le bilan de leur expérience épistolaire et s'adresse aux lecteurs de l'ouvrage qu'ils ont coproduit. Se situant nettement en rupture avec l'invocation des valeurs dans les discours politiques de droite comme de gauche, Younès brandit littéralement le mot « comprendre » comme règle majeure, capable de guider le jugement et d'orienter les comportements :

Quelle utilité cela peut-il avoir? Pourquoi m'être dévoilé à ce point? Un seul mot me vient en tête : comprendre ... Cela fait des années que je sais que tout est grillé pour nous... Alors il ne nous reste plus qu'à comprendre. Comprendre comment on en est arrivé là. [...] Mais par dessus- tout, j'aimerais que le lecteur comprenne lui aussi qu'il est d'une certaine manière responsable de tout ce merdier, que personne n'est innocent. On nous parle d'intégration, de Nation, de République, de citoyenneté... Prouvez-moi que tout ceci a un sens. [...] Qu'avez-vous fait pour votre République et votre démocratie dont vous êtes si fiers ? [...] vous avez sûrement des idéaux de gauche qui vous poussent à prôner la fraternité, l'égalité, la justice sociale ; ou bien des idéaux de droite qui vous poussent à penser que tout passe par le mérite et le travail... [...] Je n'ai aucune leçon à donner, aucune morale à faire, personne à blâmer. Je veux simplement comprendre, faire comprendre une chose : comment en est-on arrivé là ? (201).

C'est la pratique politique consistant à se prévaloir de maîtres mots consacrés par l'histoire et le consensus autour des grands textes fondateurs ou des discours idéologiques fédérateurs qui se trouve ici mise en cause avec une véhémence dont la correspondance n'a pas montré l'équivalent. "Nation», «République », " citoyenneté », « égalité ", « justice sociale », « mérite », « travail » : ces termes n’ont à aucun moment donné lieu à une contestation frontale, et, subitement, ils apparaissent comme constituant l'arrière-plan du récit et des analyses de Younès ${ }^{14}$. Valeurs rituellement invoquées, face auxquelles il convoque le mot « comprendre»: un verbe de contenu épistémique, mais qui subit un élargissement et une axiologisation éthique, invitant au retour sur soi, à la capacité de décentrement et à la prise en compte de l'altérité.

\section{Contradictions et dilemmes}

C'est sur ce fond "coopératif », que nous pouvons examiner la façon dont Younès réussit à passer d'une pratique éristique du langage à un usage plus authentiquement argumentatif destiné à exprimer les contradictions, les embarras et les dilemmes auxquels il se trouve confronté. 


\section{1. L' « affolage »}

27 Younès est beaucoup plus à l'aise à l'oral ; il évoque à de nombreuses reprises son goût pour l'« affolage », une pratique langagière ludique fondée sur l'ironie, liée au goût de juger, d'évaluer et de déstabiliser l'autre, une sorte d'éristique de quartier :

À un moment quand j'étais au lycée, y a eu une sorte de petite mode dans le quartier où tout le monde se mettait à écouter Cabrel, ça m'a toujours étonné ce truc... En tout cas c'était le prétexte à plein de vannes et d'affolages entre nous dans le bus entre pro et anti-Cabrel (74).

Ce plaisir de la dérision, qu'il revendique et pratique sans ménagement dans sa famille, Younès l'évoque à plusieurs reprises avec gourmandise.

\section{2. Désaccords et tensions}

Or, à l'opposé, Younès est amené, dans cette correspondance, à construire un ethos discursif distancié où il s'initie à l'expression des contradictions. Pour se faire comprendre (et pour se comprendre), le jeune homme doit en effet se prendre luimême pour objet de discours et intégrer la place de l'interlocuteur dans un échange. Il ne s'agit plus de produire des éclats de langage, mais de tenir une position face à un autre respecté. Cette différence dans le statut de l'interlocuteur est nettement marquée dans un courriel où il évoque son rapport aux autorités qui, bien qu'il n'ait jamais été délinquant, l'a toujours mis en délicatesse avec les institutions :

En écrivant ça, je me rends compte à quel point j'ai pu être inconscient... ; peut-être parce que personne n'était là pour me rappeler à l'ordre - je veux dire : personne de « légitime » pour moi ; [...] je peux accepter les remarques de ma femme... même de vous, sans problème... mais les autres, non... ou sinon de quelques uns... (85).

Dès le premier échange du 11 décembre, qui évoque sa visite du site Internet Homme moderne consacré à Bourdieu, Younès fait preuve de prudence ( «je ne porte pas de jugement », 13). Par la suite il lui arrive de faire un usage facétieux du vocabulaire bourdieusien (" capital social de filles », " capital culturel de quartier »), mais dans l'ensemble, il cherche à maintenir l'harmonie du dialogue sur les bases idéologiques communes (il est abonné au Monde diplomatique et à Pas Lu pas Pris, magazine satirique d'extrême gauche inspiré par les idées de Bourdieu) : la marge de désaccord est mince et pourtant elle se manifeste à plusieurs reprises.

31 Certaines pistes suggérées à Younès appellent de sa part une prise de position avec laquelle il se sent plus ou moins à l'aise. Les personnages et les événements médiatiques ayant une fonction de repérage, Beaud l'interroge sur Fadela Amara, Philippe Val, José Bové, l'apparition du mouvement " Ni putes ni soumises » né moins de deux mois après le début de leur correspondance. Soit Younès énonce franchement un désaccord avec son interlocuteur : «Je n'ai jamais aimé SOS Racisme, je ne crois pas à la pureté de leurs intentions, ce sont des nostalgiques des années 80 » (115) ; «En fait pour Philippe Val, je n'aime pas son côté bien pensant moralisateur de "gauche", ça m'énerve!» (124). Soit, plus souvent, il recourt à des formulations prudentes, marquées, sur le plan syntaxique, par le recours à des performatifs ou à des verbes d'attitude propositionnelle à valeur d'atténuation: «À propos de Ni putes ni soumises, je vous avoue mon scepticisme » (123), "Je crois que vous avez très bien compris que j'avais des positions assez particulières sur le sujet de toutes ces personnalités "beurs" (115) ; ou encore : « Pour les motivé-e-es, j’ai peur de vous choquer (je délire encore...) mais je 
crois que même eux se rendent compte que leur truc tourne en rond... » (76). Pourtant, cette modération conquise ne s'accompagne pas de suivisme, Younès ne correspondant pas à l'image idéalisée que le sociologue tend à lui renvoyer.

\section{3. L'image de soi}

32 À la lecture de l'ensemble de la correspondance, on est, en effet, frappé par le contraste entre le volontarisme de Stéphane Beaud qui invite constamment Younès à agir, à s'occuper de ses études, à passer son permis de conduire, à se rapprocher des militants associatifs de sa ville ou d'Attac ${ }^{15}$, et le pessimisme du jeune homme. Cette opposition d'humeurs (optimisme / pessimisme) intervient de manière constante comme une mesure de l'ouverture des possibles qui présuppose une évaluation de soi : Younès fluctue, passe par des phases euphoriques, mais le diagnostic pessimiste l'emporte, au moins pour ce qui concerne sa carrière. De son côté, Beaud, qui éprouve le besoin, au début, de railler la maxime "quand on veut, on peut ", perçoit la trajectoire de Younès comme un parcours inachevé, qui doit se conclure par la réussite au CAPES et le métier d'enseignant. Mais Younès récuse l'image survalorisée qui lui est renvoyée : «Vous voyez, Stéphane, je ne suis pas celui que l'on peut croire, ayant compris les mécanismes de construction sociale et qui s'en sert pour affronter l'avenir. Non, Stéphane je suis comme tous les autres de mon quartier : amer, plein d'amertume » (126). Et d'évoquer, en écho aux propos tenus auparavant par le sociologue critique, les "appels qui sonnent faux, du style "quand on veut, on peut" ", puis de s'adresser à lui directement : "Vous allez me dire qu'on fait ce qu'on veut de sa vie... et je continuerai de répondre NON, c'est faux... » (ibid.). Son acharnement à évoquer son passé " raciste » ne résulte pas seulement de son effort pour explorer son itinéraire. C'est aussi une façon de démystifier sa propre image et de refuser une identité vertueuse qui le désolidariserait de ses compagnons de galère : « on a fait de moi un raciste à force de privations, à force de dégoût des Arabes qui se transforme en haine des Français, raciste pour se dire qu'on vaut quelque chose " (17). Quand, plus tard, Beaud l'invite fermement à limiter au minimum ses rapports avec le quartier de son enfance, la tension devient vive, ce qui va conduire Younès à formuler le dilemme auquel il est confronté et qu'il ne veut pas trancher de la manière qui lui est suggérée.

\section{4. L'espace du dilemme}

33 Malpierre, c'est ce lieu, où il a vécu ses années d'adolescence et qui a constitué pour lui un repère d'identification. Younès ne cesse de le soumettre à des évaluations contradictoires - «à Berville on est connus (ou on croyait l'être) comme étant des “mecs de Malpierre" et c'est valorisant" (110) -; mais c'est pour rompre avec les dérives de l'alcool et de la drogue dont il le rendait partiellement responsable qu'il a quitté ce quartier :

Vous savez pour les jeunes comme nous, pour s'en sortir, des solutions y en a pas $50 . .$. Soit tu te casses, soit t'es un hypocrite, soit t'investis dans la religion. Moi, l'été 96, je me suis cassé du quartier et je me suis dit pour que j'arrive à décrocher sérieusement de tout ce bordel, il fallait que je rentre dans la religion (196).

Raisonnement formulé en trois branches qui se ramènent en fait à deux, l'ailleurs spatial et l'ailleurs spirituel fusionnant dans le désir de fuite. Après un enfermement de plusieurs années dans ce qu'il présente comme «l'illusion» de la religion, puis une 
stabilisation dans le couple, le souvenir des amitiés forgées dans la souffrance refait surface et conduit l'étudiant en rupture, devenu emploi-jeune, à retourner au quartier, partagé entre l'attirance et l'aversion. Et c'est du dilemme provoqué par cet « objet de valeur » ambivalent, que naît une tension avec Stéphane Beaud, début juin 2003.

Younès, après le récit d'une soirée à Malpierre marquée par un incident avec un autre jeune pris de boisson, puis un contrôle de police, conclut sur le mode ambigu qu'il adopte depuis le début: «Là, je crois vraiment que je vais bannir ce quartier et c'est dommage...» (175). Suit alors, immédiatement, un échange de courriels qui constitue un moment de clarification (avec, pour atténuer la tension latente, deux adverbes d'énonciation tampons en position initiale) :

Stéphane :

Naïvement, j'ai envie de te demander

a) que faisais-tu dans le quartier? J'ai l'impression que c'est devenu trop dur pour toi d'y aller. Tu devrais te protéger un peu et ne pas voir ça (beaucoup le font, non ?) [...]

Younès :

Paradoxalement, si je retourne au quartier de temps en temps, c'est pour décompresser, pour pallier la routine du boulot - maison. Mais en fait c'est aussi la routine le quartier. [...] Mais j'en ai besoin. (Ibid.).

La réponse du jeune homme se raidit ensuite en une dénonciation d'un comportement de fuite où le dialogisme interlocutif signale bien les points de friction : «Et puis je n'ai pas envie de faire comme certains qui ont complètement quitté le quartier et qui passent leur temps à le dénigrer. C'est peut - être une forme de protection mais c'est surtout aussi une fuite et une volonté de se cacher les yeux » (ibid.).

Se protéger et ne pas voir : c'est ce que Younès s'empresse de ne pas faire le week-end suivant, où, après plusieurs courriels très chargés émotionnellement, il retourne à Malpierre et passe la soirée avec ses amis. Dans son courrier du lundi, il met alors sous les yeux de son correspondant, ce que précisément celui-ci l'invite à " ne pas voir »: « Délires, affolages, etc... Bien sûr, y avait le bordel comme toujours... Ronde de la BAC [brigade anti-criminalité]... Rodéos, voitures volées, shit et Cie... Mais y avait une bonne ambiance » (183). Beaud interpelle le jour même Younès sur cette qualification : « Eh bien déjà peut-être réfléchir à ce que ça signifie pour toi une "bonne ambiance" et/ou une "mauvaise ambiance"... concrètement? ». Suit, dans la réponse, une évocation qui sans être provocatrice, n'élude pas les aspects sensibles :

Par rapport à l'ambiance du quartier, c'est quelque chose d'assez bizarre... [...] Mais aussi il faut être honnête en disant qu'on aime bien quand c'est un peu " chaud ». Une bonne ambiance, c'est quand les gamins foutent la merde mais sans emmerder personne. Par exemple, y a pas eu de bagarres, pas de manque de respect mais ils faisaient vraiment les dingues avec les voitures volées... ça fait du spectacle ... Les têtes à queue, les dérapages dans le boulevard, les courses-poursuites avec la BAC... toutes ces conneries... Si je dis ça à des personnes qui ne connaissent pas très bien la vie de quartier, c'est sûr que ça peut être choquant... Moi aussi ça me choque un peu car je pense aux personnes à qui ces voitures ont été volées... C'est l'ambiance d'été quoi (184).

Le caractère transgressif de ces scènes est euphémisé, mais assumé. Beaud objecte : "Mais les filles en sont exclues, non? Peut-il y avoir une bonne ambiance avec des filles?» (185). Se défendant de tout comportement macho, Younès est sur la défensive : «Vous savez, on n'est pas très à l'aise avec la mixité, vu notre éducation... » $(185)^{16}$. Échange crucial où, conscient que des limites risquent d'être franchies, il s'efforce de 
«faire voir », au-delà des stigmatisations attendues, l'intensité vécue et la capacité à surmonter une situation par le rire : " $Y$ a des types, c'est des vrais dingos, comme quoi, malgré toutes ces souffrances enfouies, on a toujours le mot pour rire». Le défi aux autorités, l'excès euphorique, la vitalité sans débordement violent : cette image d'une forme de vie excédant le choix douloureux entre une quotidienneté désespérante (dans le quartier) ou aimablement routinière (en ville, dans une vie solitaire, malgré les consolations de la vie de couple), voilà ce qui transfigure parfois le quartier en « objet de valeur " dont Younès ne réussit pas à se priver. Mais, la complicité de quartier menace la connivence avec le sociologue ( Ce que tu racontes correspond bien à ce que j'ai cru voir même si je suis toujours resté extérieur à tout ça ", 185).

À Beaud parti à Nantes pour présenter son dernier livre, Younès dans un courriel précédent écrivait : « vous avez vraiment de la chance d'avoir une vie aussi riche .... ». Tout serait dès lors en place pour la mécanique du ressentiment. Younès en a fait l'expérience dans son adolescence, à une période où, enviant les « Français ", il raconte avoir été traité par ses frères et sœurs de "raciste»: "on s'efforce de trouver des explications, très souvent superficielles, du style : "C'est la faute aux Français", "Nique sa mère, ce pays", et petit à petit, on se terre dans un brouillard, on commence par détester la France, puis on aime pas ses habitants, on idéalise le bled, on croit avoir un identité forte » (198).

\section{5. L'amour comme valeur}

Or, Younès, poursuivant son anamnèse, met au jour ce qui constitue, en plus des difficultés nées de la situation d'immigration imputables à la France, le manque affectif qu'il attribue aux « valeurs traditionnelles » de sa famille et au désarroi de ses parents débordés. Sous la pression du dilemme, surgissent des formulations qui donnent au débat axiologique qui anime la correspondance une orientation imprévue : l'amour se voit revendiqué, comme compétence, comme ouverture vers l'avenir, comme valeur non transmise par ses parents. Se rappelant les coups infligés par son père et les crises de nerfs de sa mère, il lâche: «j'aurais préféré qu'ils m'apprennent à aimer, à être aimé » (177). Younès a parfaitement conscience du caractère incongru de ses propos qui prennent complètement à rebours, non seulement les stéréotypes projetés sur les jeunes garçons arabes, mais aussi l'image qu'ils affichent pour se protéger :

C'est rare qu'un mec du quartier parle d'amour, ils vous parlent de cul, de meufs «bonnes", de sodomie... Mais jamais d'amour... Nos parents en ont fait un tabou [...] Je me répète sûrement, mais moi je veux aimer et être aimé, tout simplement, et soyez sûr que beaucoup sont dans mon cas, mais ils ne le diront pas car on ne leur a jamais appris (179).

41 Le refus du ressentiment s'était déjà exprimé non sans humour, dans un courriel précédant cette séquence :

En tout cas, moi, Ismaël, je vais tout faire pour qu'il soit super ouvert, l'inscrire dans des clubs, toutes ces conneries (je rigole), j'aimerais pas qu'il vive comme nous, plein d'aigreur, de jalousie, de haine et de rage. Je veux qu'il AIME la France tout simplement.... Moi j'ai perdu trop de temps et de sentiment avec toutes ces conneries de différences qui, il ne faut pas le nier, nous ont été imposées ! (163).

L'accent est mis désormais, entre émotions et valeurs, sur la transmission. La tâche est présentée dans toute sa difficulté, en relation avec un thème récurrent, la nécessité de "grandir » dans un environnement où la ghettoïsation retarde le passage à la phase 
adulte: "Apprendre à être un citoyen comme les autres quand on ne l'a jamais été, apprendre à aimer quand on s'est toujours senti détesté, apprendre à être un père quand...Tout ça c'est dur, c'est ça apprendre à grandir » (189). Et l'on peut vérifier l'importance de ces remarques en lisant les mots ajoutés lors de la publication de l'édition de poche: une dédicace où Younès Amrani s'adresse «à tous ceux qui n'ont pas eu la chance de pouvoir extérioriser leur souffrance, leur mal-être, leurs rancœurs et leurs regrets ${ }^{17}$, et qui se termine par ces deux lignes :

À mon épouse et à mon fils qui, j'espère, grandira

dans un pays qu'il aimera (3).

\section{Conclusion}

Comprendre et aimer. Comme on voit, c'est bien l'interaction et le contexte dans lequel ils interviennent, sur fond de valeurs implicites, qui donne à ces deux verbes courants élevés au rang de maîtres mots, leur dimension polémique, les faisant échapper aux champs de l'épistémologie ou de l'affectivité pour leur conférer une signification éthique. Cet échange épistolaire aurait pu demeurer privé ou ne constituer qu'un simple matériau de travail sociologique. Sa publication le transforme en instrument de persuasion, en un macro-acte de langage, où le professeur et l'emploi-jeune mettent sous les yeux du lecteur leur coopération et promeuvent une parole réflexive, accessible au doute et aux incertitudes. L'ethos « montré » de Younès présente en effet une tension aiguë entre une sensibilité exacerbée aux injustices et un souci ardent de se comprendre, de s'ouvrir et de changer au prix d'une vulnérabilité assumée face aux errances passées et aux ambivalences. Attitude complexe qui ne pouvait sans doute être tenue que grâce à la force morale suscitée par l'attention bienveillante et vigilante d'un interlocuteur respecté : « comme je vous ai déjà dit, je resterai toujours debout comme un vrai bonhomme» (193).

La méfiance à l'égard des valeurs institutionnelles ne résulte ici ni de l'indifférence ni du cynisme, mais de l'écart mesuré entre ce qu'elles promettent et la réalité. C'est pour pallier leur déconsidération au regard de la pratique que " comprendre » et "aimer " se voient appelés à la rescousse pour réduire la distance sociale qui est aussi une distance rhétorique. Interdiscursivité et interlocutivité conjuguent alors leurs effets : si le verbe " comprendre » a pour ainsi dire été soufflé par le discours du sociologue, c'est dans l'échange interlocutif où il se trouve enfin reconnu pour ce qu'il vaut, que Younès prend le risque étrange, vu les possibles malentendus, de s'emparer du verbe « aimer ».

\section{BIBLIOGRAPHIE}

Amrani, Younès \& Stéphane Beaud. 2005 [2004]. Pays de malheur! Un jeune de cité écrit à un sociologue, suivi de Des lecteurs nous ont écrit (Paris : La Découverte / poche)

Amossy, Ruth. 1991. Les idées reçues. Sémiologie du stéréotype (Paris : Nathan) 
Amossy, Ruth (éd.). 1999. Images de soi dans le discours. La construction de l'ethos (Lausanne :

Delachaux et Niestlé)

Angenot, Marc. 2007. Dialogues de sourds (Paris : Mille et une nuits)

Beaud, Stéphane.2002. 80\% au bac...et après ? Les enfants de la démocratisation scolaire (Paris : La Découverte)

Beaud, Stéphane \& Michel Pialoux. 2006. « Des “marcheurs” de 1983 aux “émeutiers” de 2005. Deux générations sociales d'enfants d'immigrés », Annales. Histoire, Sciences sociales 61-4, 809-844

Charaudeau, Patrick. 1992. Grammaire du sens et de l'expression (Paris : Hachette)

Charaudeau, Patrick. 2008. Entre populisme et peopolisme. Comment Sarkozy a gagné! (Paris : Vuibert)

Doury, Marianne. 2000. « Argumentation et mise en voix. Les discours quotidiens sur l'immigration », Bondi, Maria \& Sorin Stati (éds.), Dialogue analysis 2000, Selected papers from the 10th IADA Anniversary Conference (Bologne : Niemeyer), 173-183

Ducrot, Oswald. 1972. Dire et ne pas dire (Paris : Hermann)

Guerrini, Jean-Claude \& Edmond Majcherczak. 1999. L'Argumentation au pluriel. Polyphonie, valeurs, points de vue (Lyon : Presses Universitaires de Lyon)

Kerbrat-Orecchioni, Catherine. 1980. L'énonciation. De la subjectivité dans le discours. (Paris : Colin)

Kerbrat-Orecchioni, Catherine. 2005. Le discours en interaction (Paris : Colin)

Maingueneau, Dominique. 2007. Analyser les textes de communication (Paris : Colin)

Perelman, Chaïm \& Lucie Olbrechts-Tyteka. 1988 [1958]. Traité de l'argumentation. La nouvelle rhétorique (Bruxelles : Editions de l'Université de Bruxelles)

Plantin, Christian. 1998. "Les raisons des émotions », Bondi, M. (éd.). Forms of argumentative discourse / Per un'analisi linguistica dell' argomentare (Bologne : CLUEB), 3-50

Plantin, Christian. 2005. L'argumentation (Paris : PUF)

Siess, Jürgen \& Séverine Hutin (éds). 2005. Le rapport de places dans l'épistolaire, Semen $\mathrm{n}^{\circ} 20$.

Zaccaï-Reyners, Nathalie (éd.). 2003. Explication-Compréhension : Regards sur les sources et l'actualité d'une controverse épistémologique (Bruxelles : Editions de l'Université Libre de Bruxelles)

\section{NOTES}

1. L'ouvrage a paru à La Découverte en 2004. La $2^{\mathrm{e}}$ édition à La Découverte/poche (2005) comporte un ajout : "Des lecteurs nous ont écrit ».

2. Sous forme d'articles ou de notes de lecture, notamment dans Le Monde (Michel Samson, Le beur et le sociologue, 3 décembre 2004, p. 24), La Quinzaine littéraire (Numa Murard, «Une sociologie virtuelle?» $\mathrm{n}^{\circ}$ 892, 16 janvier 2005, p. 16), Esprit (Michel Kokoreff, février 2005. pp. 219-223), Espacetemps. Cahiers (Fabrice Ripoll. «La double distance. Note de lecture sur une socio-analyse épistolaire »). http://espacestemps.revues.org. Consulté le 5 janvier 2009.

3. Le mot "axiologique» est employé ici au sens large, comme adjectif correspondant au substantif valeur (de axios qui désigne ce qui vaut, ce qui a de la valeur, ce qui est digne, ce qui vaut la peine : Dictionnaire grec- français d'A. Bailly).

4. La question de savoir quelles sont les orientations principales de l'évaluation est en discussion depuis l'Antiquité. Patrick Charaudeau (1995) retient les « domaines » suivants : le domaine de la Vérité, le domaine de l'Esthétique, le domaine de l'Éthique, le domaine de l'Hédonique, le 
domaine du Pragmatique. Pour ma part, en évitant le recours aux majuscules et en substituant le mot axe au mot domaine (qui ne permet guère de rendre compte des phénomènes de tension entre valeurs), je parlerais plutôt d'axes de valorisation ou de dévalorisation esthétique, éthique, hédonique, pragmatique et épistémique.

5. Cité dans Perelman \& Olbrechts-Tyteca (1988:171).

6. L'ouvrage ne nous renseigne guère sur la nature de ces entretiens peu évoqués dans la correspondance. Dans ses courriels, Beaud ne prétend d'ailleurs pas à la rigueur méthodologique qu'il mobilise ailleurs. La sympathie pour son informateur l'emporte nettement sur la simple empathie nécessaire à l'enquête; les motifs personnels déterminant le thème de recherche s'expriment ici sans détour : «Les patrons français sont allés chercher dans leur cambrousse des paysans pauvres et analphabètes et il faudrait que leurs enfants nés en France n'en subissent pas les conséquences... Moi, c'est cette hypocrisie sociale qui m'écœure » (150).

7. Graphie d'insistance délibérée.

8. L'inflexion engendrée par ce changement de cadre interlocutif est difficile à repérer. Elle ne se fait nettement sentir que dans les tout derniers courriels.

9. Beaud tutuoiera Younès après sa « montée » à Paris, mais sans réciprocité.

10. En 1993, Beaud a participé à l'entreprise collective de La Misère du monde coordonnée par Pierre Bourdieu. Il a publié chez Fayard, avec Michel Pialoux, Retour sur la condition ouvrière (Fayard, 1999).

11. Younès insiste sur le rôle de refuge qu'a eu pour lui la bibliothèque : le livre, valorisé par souci de « distinction » (note-t-il ironiquement), l'a toujours accompagné (23).

12. Les auteurs, se référant eux-mêmes à Dupréel, visent dans ce passage le « vague » qui marque les « valeurs universelles."

13. Voir sur ce point voir Zaccaï-Reyners 2003.

14. À propos de SOS Racisme, Younès déclarait déjà : «Les mecs en ont plus rien à foutre de ces conneries de respect et de tolérance puisqu'ils ne sont respectés nulle part » (115).

15. Association pour la Taxation des Transactions financières et l'Action citoyenne, créée en France en 1998

16. Mais à une question de Beaud concernant Ni Putes ni soumises, Younès avait fait remarquer quelques mois avant: "Et puis les filles ont le beau rôle dans l'histoire, on accuse toujours les mêmes : les jeunes de quartiers, ah ceux-là ont toutes les tares du monde : machos, "sauvageons", irrespectueux, touristes à l'école, bons à rien, délinquants, j'en passe et des meilleures » (123).

17. Le titre Pays de malheur! n'est pas la reprise d'un propos de Younès, mais d'un de ses amis dont il décrit la détresse au début de la correspondance (29).

\section{RÉSUMÉS}

La recherche d'un accord sur les valeurs permet au locuteur de définir et orienter son point de vue. Mais loin d'être toujours immédiatement disponible et clairement identifié, le répertoire axiologique peut donner lieu à une explicitation ou à une élaboration progressive : la relation épistolaire fournit un cadre coopératif propice à une telle démarche. Cet article porte sur la correspondance électronique privée qu'ont entretenue, en 2002/2003, un jeune travaillant dans une bibliothèque municipale de la banlieue lyonnaise et un sociologue renommé, correspondance 
qui fut ensuite publiée et promue au rang de témoignage sur les souffrances, les dilemmes et les aspirations des «jeunes de quartier». L'étude de ce document, menée dans une perspective pragmatique, porte sur l'enchevêtrement des ressources verbales permettant de formuler une position axiologique et des mécanismes de valorisation / dévalorisation affectant les différents éléments de la communication (participants, objets, comportements et pratiques langagières).

The search of an agreement over values leads the speaker to define his standpoint and to orient his discourse. But, far from being clearly identified and immediately available, the axiological data are often left implicite. In front of a claim for justification, they may be explicited or built up gradually: the epistolary genre draws on a suitable cooperative frame to explore such a situation. This paper deals with a private electronic correspondence (2002- 2003) between a young man working in a public library near Lyon and a well-known sociologist, which has been published later on as an account of the situation of the stigmatized, suburban youth (sufferings, dilemmas and wishes). What is mainly examined here, from a pragmatic point of view, is the intertwining relations of the axiological verbal ressources and the valuation of the different elements of the communication (participants, objects, behavior, and verbal attitudes).

\section{INDEX}

Mots-clés : axiologique, dilemme, interaction, valeur, valorisation

Keywords : axiological, dilemma, interaction, valuation, value

\section{AUTEUR}

\section{JEAN-CLAUDE GUERRINI}

Université Lumière Lyon 2 